\title{
Nonlinear damped standing slow waves in hot coronal magnetic loops
}

\author{
M. S. Ruderman \\ 1 Solar Physics and Space Plasma Research Centre $\left(\mathrm{SP}^{2} \mathrm{RC}\right)$, University of Sheffield, Hicks Building, Hounsfield Road, \\ Sheffield S3 7RH, UK \\ 2 Space Research Institute (IKI), Russian Academy of Sciences, 117997 Moscow, Russia \\ e-mail: m.s.ruderman@sheffield.ac.uk
}

Received 27 January 2013 / Accepted 19 March 2013

\begin{abstract}
We study the nonlinear standing slow waves in coronal magnetic loops. We assume that the wave amplitude is sufficiently small and use it as a small parameter in the asymptotic expansions. In addition, we assume that the wave damping is sufficiently slow, which enables us to introduce the "slow" time related to the evolution of the wave amplitude and shape. We show that, in the leading-order approximation, the nonlinear standing wave is a superposition of two identical nonlinear waves propagating in the opposite directions. The two waves are governed by the Burgers equation. Using the Cole-Hopfe substitution, we obtain an analytical solution describing a standing wave that has the form of the linear fundamental eigenmode at the initial moment of time. This solution is used for the parametric study of nonlinear standing waves. In particular, the effect of nonlinearity on the oscillation damping time is investigated.
\end{abstract}

Key words. magnetohydrodynamics (MHD) - plasmas - waves - methods: analytical

\section{Introduction}

Standing waves in hot $(T \gtrsim 6 \mathrm{MK})$ coronal loops were observed by the Solar Ultraviolet Measurements of Emitted Radiation (SUMER) spectrometer onboard the SOHO spacecraft (Kliem et al. 2002; Wang et al. 2002, 2003a,b). Observations of 17 events revealed that the oscillation periods ranged from 11 to $31 \mathrm{~min}$, and the decay time from 5.5 to 29 min. Ofman \& Wang (2002) interpreted these oscillations as slow standing waves. The velocity amplitude in these oscillations was up to one quarter of the phase speed, so at least some of them were moderately nonlinear. Ofman \& Wang (2002) modelled these oscillations numerically and found that the main damping mechanism causing their damping is thermal conduction. They also found that the damping prevents strong nonlinear steepening and shock formation. For a recent review of standing slow waves in coronal loops see Wang (2011).

In their modelling Ofman \& Wang (2002) used the direct numerical solution of the one-dimensional non-stationary nonlinear equations describing the motion of compressible fluid with the account of viscosity and thermal conduction. They carried out the modelling only for a restricted range of parameters. More recently, a similar numerical modelling has been carried out by Mendoza-Briceño et al. (2004), Sigalotti et al. (2007), and Verwichte et al. (2008) for a wider range of coronal loop parameters and initial perturbation amplitude.

In this paper we aim to present a semi-analytical study of slow standing waves in hot coronal loops. Our analysis is based on the fact that the oscillation amplitude is quite moderate, so the dimensionless amplitude can be used as a small parameter in the asymptotic expansion. As a result we derive a simple differential equation describing the perturbation evolution with time. This enables us to carry out the parametric study of oscillations for a wide range of parameters.

The paper is organized as follows. In the next section we formulate the problem, discuss the main assumptions, and present the governing equations. In Sect. 3 we derive the nonlinear equation describing the time-evolution of the velocity in the slow standing wave. In Sect. 4 we obtain the analytic solution describing the evolution with time of the perturbation corresponding to the fundamental mode of slow standing waves. We then use this solution for the parametric study of the nonlinear damping of slow standing waves. Section 5 contains the summary of the obtained results and our conclusions.

\section{Problem formulation and main assumptions}

We study slow standing waves in hot coronal loops with the temperature $T \gtrsim 6 \mathrm{MK}$. The atmospheric scale height in these hot loops is larger than $300 \mathrm{Mm}$. This implies that, in these loops, we can safely neglect the density variation if the height of the loop apex point is smaller than or of the order of $100 \mathrm{Mm}$. The plasma number density in hot loops usually does not exceed $10^{15} \mathrm{~m}^{-3}$. Then, for $T \leq 10 \mathrm{MK}$, the plasma pressure does not exceed $0.3 \mathrm{Nm}^{-2}$. The magnetic pressure is equal to $B^{2} / 2 \mu_{0}$, where $B$ is the magnetic field magnitude and $\mu_{0} \approx 1.26 \times 10^{-6} \mathrm{H} \mathrm{m}^{-1}$ is the magnetic permeability of free space. Then we easily find that the plasma beta is smaller than 0.1 when $B \gtrsim 10^{-3}$ Tesla $=10 \mathrm{G}$. Since this condition is usually satisfied in the coronal loops, we can use the low-beta plasma approximation when studying the slow waves in hot coronal loops. This implies that we can neglect the magnetic field perturbation. If, in addition, we neglect the variation of the loop cross-section and the equilibrium quantities in the directions perpendicular to the loop axis, and the 
loop curvature, then we can describe the slow waves by onedimensional hydrodynamic equations (Priest 1982; Goedbloed \& Poedts 2004)

$\frac{\partial \rho}{\partial t}+\frac{\partial(\rho u)}{\partial x}=0$

$\frac{\partial u}{\partial t}+u \frac{\partial u}{\partial x}=-\frac{1}{\rho} \frac{\partial p}{\partial x}+\frac{1}{\rho} \frac{\partial}{\partial x} \rho v \frac{\partial u}{\partial x}$,

$\frac{\partial T}{\partial t}+u \frac{\partial T}{\partial x}+(\gamma-1) T \frac{\partial u}{\partial x}=\frac{\partial}{\partial x} \kappa \frac{\partial T}{\partial x}$,

$p=\frac{k_{\mathrm{B}}}{m} \rho T$.

Here $u$ is the velocity, $\rho$ the density, $p$ the pressure, $T$ the temperature, $\gamma$ the ratio of specific heats, $k_{\mathrm{B}}$ the Boltzmann constant, and $m$ the mean mass for a particle ( $m \approx 0.6 m_{\mathrm{p}}$ in the solar corona, where $m_{\mathrm{p}}$ is the proton mass). The coefficients $v$ and $\kappa$ are defined by

$v=\frac{4 \eta_{0}}{3 \rho}, \quad \kappa=\frac{(\gamma-1) m k_{\|}}{\rho k_{\mathrm{B}}}$,

where $k_{\mathrm{B}}$ is the Boltzmann constant, $\eta_{0}$ the first viscosity coefficient in the Braginskii's expression for the viscosity tensor, and $k_{\|}$the thermal conductivity parallel to the magnetic field. These latter two quantities are given by (Braginskii 1965)

$\eta_{0} \approx n k_{\mathrm{B}} T \tau_{\mathrm{p}}$,

and (Spitzer 1962; Priest 1982)

$k_{\|} \approx 10^{-11} T^{5 / 2} \mathrm{~W} \mathrm{~m}^{-1} \mathrm{~K}^{-1}$,

where $n=\rho / m_{\mathrm{p}}$ is the number density and $\tau_{\mathrm{p}}$ the proton collision time. It is given by the approximate expression

$\tau_{\mathrm{p}} \approx 1.66 \times 10^{7} \frac{T^{3 / 2}}{n \ln \Lambda} \mathrm{s}$

where $\Lambda$ is the Coulomb logarithm and the number density $n$ is measured in $\mathrm{m}^{-3}$. Fot typical conditions in hot loops $\ln \Lambda \approx 20$, and $\tau_{\mathrm{p}}$ is approximately between 10 and $25 \mathrm{~s}$.

The system of Eqs. (1)-(4) is essentially the same as the one used by Ofman \& Wang (2002). The only difference is that we neglected the term describing the viscous heating in the energy Eq. (4). The reason for this is that this term is quadratic with respect to $u$, while, in what follows, we linearise the dissipative terms. The system of Eqs. (1)-(4) has to be supplemented with the boundary conditions. We assume that the magnetic loop is frozen in the dense photosphere, so that

$u=0 \quad$ at $\quad x=0, L$,

where $L$ is the loop length.

The system of Eqs. (1)-(4) with the boundary conditions (9) is used in the next section to derive the equation governing the evolution of the velocity with time in a slow standing wave.

\section{Derivation of the governing equation for the oscillation velocity}

In this section we derive the equation governing the evolution of the oscillation velocity with time. If we neglect the nonlinear and dissipative terms in Eqs. (1)-(4), this system of equations admits solutions describing periodic standing slow waves in the loop. We assume that the nonlinear and dissipative terms are small and only cause a slow evolution of perturbations. This assumption enables us to use the method of two scales with the "fast time" related to the quasi-periodic oscillations and "slow" time related to slow evolution of oscillations (e.g. Bender \& Orszag 1978). Before starting the derivation we comment on the criterion of applicability of the two-scale method. When studying damped oscillations it is usually assumed that these oscillations can be considered as slowly evolving and the two-scale method can be applied if the characteristic damping time is much longer than the oscillation period. However, this is not completely correct. In the linear theory a typical function describing the damped oscillations is $\mathrm{e}^{-\gamma t} \sin \omega t$. The characteristic damping time is $1 / \gamma$. It is also clear that the characteristic time related to the oscillation is $1 / \omega$ rather than the oscillation period $2 \pi / \omega$. This implies that the criterion for the applicability of the two-scale method is $\gamma / \omega \ll 1$. We see that even when the characteristic damping time is equal to the oscillation period, we have $\gamma / \omega<0.2$ and the use of the two-scale method is perfectly correct. Since, in accordance with observations, the characteristic damping time of the slow standing waves in hot coronal loops is longer than or of the order of the oscillation period, we can use the two-scale method to study these oscillations. Then we use the asymptotic method based on the expansions with respect to the small amplitude of oscillations. To complete this introductory discussion we mention that a similar method of asymptotic expansions was previously successfully applied by Gusev $(1984 b$,a) to study the steady state of oscillation of driven standing nonlinear waves in a finite-length wave guide, and by Ruderman \& Nocera (1998) to study the steady state of driven Alfvén oscillations in a magnetic cavity.

In what follows we assume that the dissipation is weak and introduce the scaled coefficients at the dissipative terms, $\bar{v}=\epsilon^{-1} \nu$ and $\bar{\kappa}=\epsilon^{-1} \kappa$, where $\epsilon \ll 1$. We also assume that the oscillation amplitude is sufficiently small and neglect the dependence of $v$ and $\kappa$ on $T$. Hence, in what follows, $v$ and $\kappa$ are constant. Finally we introduce the "slow" time $t_{1}=\epsilon t$. Then Eqs. (1)-(3) are transformed to

$$
\begin{aligned}
& \frac{\partial \rho}{\partial t}+\frac{\partial(\rho u)}{\partial x}=-\epsilon \frac{\partial \rho}{\partial t_{1}} \\
& \frac{\partial u}{\partial t}+u \frac{\partial u}{\partial x}+\frac{1}{\rho} \frac{\partial p}{\partial x}=-\epsilon \frac{\partial u}{\partial t_{1}}+\epsilon \bar{v} \frac{\partial^{2} u}{\partial x^{2}}, \\
& \frac{\partial T}{\partial t}+u \frac{\partial T}{\partial x}+(\gamma-1) T \frac{\partial u}{\partial x}=-\epsilon \frac{\partial T}{\partial t_{1}}+\epsilon \bar{\kappa} \frac{\partial^{2} T}{\partial x^{2}}
\end{aligned}
$$

Equation (4) remains unchanged. We look for the solution to the system of Eqs. (4) and (10)-(12) with the boundary conditions (9) in the form of expansions

$f=f_{0}+\epsilon f_{1}+\epsilon^{2} f_{3}+\ldots$

where $f$ represents any of the quantities $u, \rho, p$ and $T$. The first term, $f_{0}$, corresponds to the unperturbed state, so $f_{0}=$ const. Note that $u_{0}=0$.

\subsection{First-order approximation}

In the first-order approximation we substitute the expansion (13) in Eqs. (4) and (10)-(12), and the boundary condition (9) and 
collect the terms proportional to $\epsilon$. As a result we obtain

$$
\begin{aligned}
& \frac{\partial \rho_{1}}{\partial t}+\rho_{0} \frac{\partial u_{1}}{\partial x}=0, \\
& \frac{\partial u_{1}}{\partial t}+\frac{1}{\rho_{0}} \frac{\partial p_{1}}{\partial x}=0, \\
& \frac{\partial T_{1}}{\partial t}+(\gamma-1) T_{0} \frac{\partial u_{1}}{\partial x}=0, \\
& p_{1}=\frac{k_{\mathrm{B}}}{m}\left(\rho_{0} T_{1}+T_{0} \rho_{1}\right), \\
& u_{1}=0 \text { at } \quad x=0, L .
\end{aligned}
$$

Eliminating perturbations of all variables in favour of $u_{1}$, we obtain

$\frac{\partial^{2} u_{1}}{\partial t^{2}}-c_{\mathrm{s}}^{2} \frac{\partial^{2} u_{1}}{\partial x^{2}}=0$

where $c_{\mathrm{s}}=\left(\gamma p_{0} / \rho_{0}\right)^{1 / 2}$ is the adiabatic sound speed. When deriving this equation we have used he relation $p_{0}=k_{\mathrm{B}} \rho_{0} T_{0} / m$ that follows from Eq. (4). The general solution to Eq. (19) can be written as

$u_{1}=c_{\mathrm{s}}[f(\xi)+g(\eta)], \quad \xi=\omega\left(t-\frac{x}{c_{\mathrm{s}}}\right), \quad \eta=\omega\left(t+\frac{x}{c_{\mathrm{s}}}\right)$,

where, at present, $f$ and $g$ are arbitrary functions, and $\omega$ is an arbitrary constant with the dimension $\mathrm{s}^{-1}$. Note that $f$ and $g$ also depend on $t_{1}$, however, at present, we do not write this argument explicitly. Substituting Eq. (20) in Eq. (18), we obtain

$f(\omega t)+g(\omega t)=0, \quad f\left(\omega\left(t-L / c_{\mathrm{s}}\right)\right)+g\left(\omega\left(t+L / c_{\mathrm{s}}\right)\right)=0$.

It follows from these equations that $g(y)=-f(y)$, and $f(y)$ is a function with the period equal to $2 \omega L / c_{\mathrm{S}}$. It is convenient to define $\omega$ such that the period of function $f(y)$ is equal to $2 \pi$. In this case $\omega=\pi c_{\mathrm{s}} / L$. Summarizing, we obtain that

$u_{1}=c_{\mathrm{s}}[f(\xi)-f(\eta)]$,

where $f$ is a $2 \pi$-periodic function. Now it follows from Eqs. (14), (15), and (17) that

$\rho_{1}=\rho_{0}[f(\xi)+f(\eta)], \quad p_{1}=\rho_{0} c_{\mathrm{s}}^{2}[f(\xi)+f(\eta)]$,

$T_{1}=(\gamma-1) T_{0}[f(\xi)+f(\eta)]$

\subsection{Second-order approximation}

In the second-order approximation we collect the terms proportional to $\epsilon^{2}$ in Eqs. (4) and (10)-(12), and the boundary condition (9). Then, using Eqs. (22)-(24), we obtain

$$
\begin{aligned}
\frac{\partial \rho_{2}}{\partial t}+\rho_{0} \frac{\partial u_{2}}{\partial x}= & 2 \omega \rho_{0}\left(f_{-} f_{-}^{\prime}+f_{+} f_{+}^{\prime}\right)-\rho_{0}\left(\frac{\partial f_{-}}{\partial t_{1}}+\frac{\partial f_{+}}{\partial t_{1}}\right), \\
\frac{\partial u_{2}}{\partial t}+\frac{1}{\rho_{0}} \frac{\partial p_{2}}{\partial x}= & 2 \omega c_{\mathrm{s}}\left(f_{-} f_{+}^{\prime}-f_{+} f_{-}^{\prime}\right) \\
& -c_{\mathrm{s}}\left(\frac{\partial f_{-}}{\partial t_{1}}-\frac{\partial f_{+}}{\partial t_{1}}\right)+\frac{\bar{v} \omega^{2}}{c_{\mathrm{s}}}\left(f_{-}^{\prime \prime}-f_{+}^{\prime \prime}\right),
\end{aligned}
$$

$$
\begin{aligned}
\frac{\partial T_{2}}{\partial t}+(\gamma-1) & T_{0} \frac{\partial u_{2}}{\partial x}=(\gamma-1) \omega T_{0}\left[\gamma\left(f_{-} f_{-}^{\prime}+f_{+} f_{+}^{\prime}\right)\right. \\
& \left.-(2-\gamma)\left(f_{-} f_{+}^{\prime}+f_{+} f_{-}^{\prime}\right)\right] \\
& -T_{0}\left(\frac{\partial f_{-}}{\partial t_{1}}+\frac{\partial f_{+}}{\partial t_{1}}\right)+\frac{(\gamma-1) \bar{\kappa} \omega^{2} T_{0}}{c_{\mathrm{s}}^{2}}\left(f_{-}^{\prime \prime}+f_{+}^{\prime \prime}\right),
\end{aligned}
$$$$
p_{2}-\frac{k_{\mathrm{B}}}{m}\left(\rho_{0} T_{2}+T_{0} \rho_{2}\right)=p_{0}(\gamma-1)\left(f_{-}+f_{+}\right)^{2},
$$

$u_{2}=0 \quad$ at $\quad x=0, L$, where $f_{-}=f\left(\xi, t_{1}\right), f_{+}=f\left(\eta, t_{1}\right)$, and the prime denotes the partial derivative of $f\left(y, t_{1}\right)$ with respect to $y$. Eliminating $\rho_{2}, p_{2}$, and $T_{2}$ from Eqs. (25)-(28), we obtain the equation for $u_{2}$ :

$$
\begin{aligned}
& \frac{\partial^{2} u_{2}}{\partial t^{2}}-c_{\mathrm{s}}^{2} \frac{\partial^{2} u_{2}}{\partial x^{2}}=\frac{\omega^{3}[\gamma \bar{v}+(\gamma-1) \bar{\kappa}]}{\gamma c_{\mathrm{s}}}\left(f_{-}^{\prime \prime \prime}-f_{+}^{\prime \prime \prime}\right) \\
& -2 \omega c_{\mathrm{s}}\left(\frac{\partial f_{-}^{\prime}}{\partial t_{1}}-\frac{\partial f_{+}^{\prime}}{\partial t_{1}}\right)+\omega^{2} c_{\mathrm{s}}\left[( \gamma + 1 ) \left(f_{-}^{\prime 2}-f_{+}^{\prime 2}\right.\right. \\
& \left.\left.+f_{-} f_{-}^{\prime \prime}-f_{+} f_{+}^{\prime \prime}\right)+(3-\gamma)\left(f_{-} f_{+}^{\prime \prime}-f_{+} f_{-}^{\prime \prime}\right)\right] \text {. }
\end{aligned}
$$

We introduce the Fourier series

$u_{2}\left(t, x, t_{1}\right)=\sum_{n=-\infty}^{\infty} U_{n}\left(x, t_{1}\right) \mathrm{e}^{\mathrm{i} n \omega t}$,

$f\left(y, t_{1}\right)=\sum_{n=-\infty}^{\infty} f_{n}\left(t_{1}\right) \mathrm{e}^{\mathrm{i} n y}$.

where $U_{-n}=U_{n}^{*}, f_{-n}=f_{n}^{*}$, and the asterisk denotes the complex conjugate. Without loss of generality we can always assume that $f_{0}=0$. Substituting these expressions in Eq. (30), and using the identity

$$
\begin{aligned}
f(y) g(y) & =\sum_{n=-\infty}^{\infty} \mathrm{e}^{\mathrm{i} n y} \sum_{m=-\infty}^{\infty} f_{m} g_{n-m}, \\
f(\xi) g(\eta) & =\sum_{n=-\infty}^{\infty} \mathrm{e}^{\mathrm{i} n \omega t} \sum_{m=-\infty}^{\infty} f_{m} g_{n-m} \mathrm{e}^{\mathrm{i} \pi x(n-2 m) / L} \\
& =\sum_{n=-\infty}^{\infty} \mathrm{e}^{\mathrm{i} n \omega t} \sum_{m=-\infty}^{\infty} f_{n-m} g_{m} \mathrm{e}^{\mathrm{i} \pi x(2 m-n) / L},
\end{aligned}
$$

valid for real functions $f$ and $g$, we obtain

$$
\begin{aligned}
\frac{\partial^{2} U_{n}}{\partial x^{2}} & +\frac{\pi^{2} n^{2}}{L^{2}} U_{n}=\frac{2 \omega^{2}}{c_{\mathrm{s}}}\left[\left(\frac{\omega n^{3}[\gamma \bar{v}+(\gamma-1) \bar{\kappa}]}{\gamma c_{\mathrm{s}}^{2}} f_{n}\right.\right. \\
& \left.+\frac{2 n}{\omega} \frac{\partial f_{n}}{\partial t_{1}}-\mathrm{i} n(\gamma+1) \sum_{m=-\infty}^{\infty} m f_{m} f_{n-m}\right) \sin \frac{\pi n x}{L} \\
& \left.-\mathrm{i}(3-\gamma) \gamma \sum_{m=-\infty}^{\infty} m^{2} f_{m} f_{n-m} \sin \frac{\pi(n-2 m) x}{L}\right]
\end{aligned}
$$

It follows from Eq. (9) that $U_{n}$ satisfies the boundary conditions

$U_{n}=0 \quad$ at $\quad x=0, L$.

The homogeneous counterpart of Eq. (35) together with the boundary conditions (36) constitute the Sturm-Liouville problem. It has a non-trivial solution $U_{n}=\sin (\pi n x / L)$. This implies that Eq. (35) has a solution satisfying the boundary conditions (36) only if the right-hand side of it satisfies the compatibility condition. This compatibility condition is the condition that it is orthogonal to the function $\sin (\pi n x / L)$, i.e. that the integral of the product of the right-hand side and the function $\sin (\pi n x / L)$ over the interval $[0, L]$ is equal to zero. In the last sum on the right-hand side of Eq. (35) only two terms, one proportional to $\sin (\pi n x / L)$ and the other proportional to $-\sin (\pi n x / L)$ can give non-zero contributions in this integral. However, due to the assumption that $f_{0}=0$, these two terms are identically zero. Hence, the last sum on the right-hand side of Eq. (35) does not contribute in the integral. It is easy to show that this conclusion remains correct even if we do not assume 
that $f_{0}=0$. Then we immediately obtain that the compatibility condition is

$\frac{\partial f_{n}}{\partial t_{1}}-\frac{\mathrm{i} \omega(\gamma+1)}{2} \sum_{m=-\infty}^{\infty} m f_{m} f_{n-m}+\frac{\omega^{2} n^{2}[\gamma \bar{v}+(\gamma-1) \bar{\kappa}]}{2 \gamma c_{\mathrm{s}}^{2}} f_{n}=0$

Multiplying this equation by $\mathrm{e}^{\mathrm{i} n y}$ and taking the sum with respect to $n$ from $-\infty$ to $\infty$ we obtain

$\frac{\partial f}{\partial \tau}-2 \lambda f \frac{\partial f}{\partial y}-\frac{\partial^{2} f}{\partial y^{2}}=0$

where $\tau=t / t_{\mathrm{dl}}$,

$t_{\mathrm{dl}}=\frac{2 \gamma L^{2}}{\pi^{2}[\gamma \nu+(\gamma-1) \kappa]}, \quad \lambda=\frac{\epsilon \gamma(\gamma+1) c_{\mathrm{s}} L}{2 \pi[\gamma \nu+(\gamma-1) \kappa]}$.

Here $t_{\mathrm{dl}}$ is the damping time given by the linear theory. We see that the function $f$ satisfies the Burgers Eq. (38). The Burgers equation is one of the basic equations in nonlinear acoustics (e.g. Burgers 1948; Whitham 1974; Rudenko \& Solujan 1977). In the leading-order approximation with respect to the small parameter $\epsilon$ the plasma velocity, density, pressure, and temperature are given by Eqs. (22)-(24). Hence, in the leading-order approximation, the nonlinear standing wave is a superposition of two identical nonlinear waves propagating in the opposite directions. The evolution of each of these waves is governed by the Burgers Eq. (38).

\section{Nonlinear damping of slow waves}

In this section we use the results obtained in the previous section to study the damping of nonlinear standing waves. We assume that, at the initial moment of time, the function $f$ is given by

$f(y, 0)=-\sin y$.

This implies that in the leading-order approximation

$u=2 \epsilon c_{\mathrm{S}} \sin \frac{\pi x}{L}, \quad \rho=\rho_{0}, \quad p=p_{0}, \quad T=T_{0}$ at $t=0$.

We see that the initial wave amplitude is $2 \epsilon c_{\mathrm{s}}$. Hence, $2 \epsilon$ is the perturbation Mach number at the initial moment of time. To obtain the $2 \pi$-periodic solution of Eq. (38) satisfying the initial condition (40), we use the famous Cole-Hopf (Cole 1951; Hopf 1950) substitution

$f=\frac{1}{\lambda h} \frac{\partial h}{\partial y}$.

Substituting this expression in Eq. (38) yields

$\frac{\partial h}{\partial \tau}=\frac{\partial^{2} h}{\partial y^{2}}$

The function $h$ has to be $2 \pi$-periodic. It follows from Eqs. (40) and (42) that $h$ has to satisfy the initial condition

$h(y, 0)=\mathrm{e}^{\lambda \cos y}$.

The general $2 \pi$-periodic solution to Eq. (43) is

$h(y, \tau)=a_{0}+\sum_{n=1}^{\infty} \mathrm{e}^{-n^{2} \tau}\left[a_{n} \cos (n y)+b_{n} \sin (n y)\right]$.
Using the formulae (e.g. Abramowitz \& Stegun 1964)

$I_{n}(z)=\frac{1}{\pi} \int_{0}^{\pi} \mathrm{e}^{z \cos y} \cos (n y) \mathrm{d} y$,

where $n=0,1, \ldots$ and $I_{n}$ is the modified Bessel function of the first kind and order $n$, we obtain the Fourier expansion

$\mathrm{e}^{\lambda \cos y}=I_{0}(\lambda)+2 \sum_{n=1}^{\infty} I_{n}(\lambda) \cos (n y)$.

It follows from Eq. (44), and the comparison of the Fourier expansion (45) calculated at $\tau=0$ and the Fourier expansion (47) that

$a_{0}=I_{0}(\lambda), \quad a_{n}=2 I_{n}(\lambda), \quad b_{n}=0, \quad n=1,2, \ldots$

Substituting these expressions in Eq. (45), we eventually arrive at

$h(y, \tau)=I_{0}(\lambda)+2 \sum_{n=1}^{\infty} I_{n}(\lambda) \mathrm{e}^{-n^{2} \tau} \cos (n y)$.

This equation together with Eq. (42) define the $2 \pi$-periodic solution to Eq. (38) satisfying the initial condition (40). We see that, in the dimensionless variables, the evolution of the initial perturbation depends on one dimensionless parameter $\lambda$ only. Using Eqs. (4)-(8), we obtain the approximate expression

$\lambda \approx 5 \times 10^{-10} \epsilon n_{0} L T_{0}^{-2}$,

$n_{0}$ is in $\mathrm{m}^{-3}, L$ is in $\mathrm{m}$, and $T_{0}$ is in Kelvin, and we have taken the Coulomb logarithm $\ln \Lambda=20$.

The parameter $\lambda$ can vary in a quite large limits. For instance, in the example considered by Ofman \& Wang (2002) the loop parameters are as follows: $L \approx 400 \mathrm{Mm}, n=5 \times 10^{14} \mathrm{~m}^{-3}$ and $T=6.3 \mathrm{MK}$. The initial perturbation Mach number is about 0.2 , so $\epsilon \approx 0.1$. Substituting these numbers in Eq. (50), we obtain $\lambda \approx 0.25$. If we take higher values of $\epsilon$ and/or $n$, we can obtain the value of $\lambda$ exceeding unity. However, for typical hot loop parameters and moderate Mach numbers of the initial amplitude we can expect that $\lambda$ is sufficiently smaller than unity. In that case we can obtain the approximate solution using the expansion in the power series with respect to $\lambda$. Using the expansions of the modified Bessel functions in the power series (e.g. Abramowitz \& Stegun 1964), we obtain from Eq. (49)

$$
\begin{aligned}
h(y, \tau)= & 1+\frac{\lambda^{2}}{4}+\lambda\left(1+\frac{\lambda^{2}}{8}\right) \mathrm{e}^{-\tau} \cos y \\
& +\frac{\lambda^{2}}{4} \mathrm{e}^{-4 \tau} \cos (2 y)+\frac{\lambda^{3}}{24} \mathrm{e}^{-9 \tau} \cos (3 y)+O\left(\lambda^{4}\right) .
\end{aligned}
$$

Substituting this expression in Eq. (42) yields

$$
\begin{aligned}
f(y, \tau)= & \mathrm{e}^{-\tau}\left[1-\frac{\lambda^{2}}{8}\left(1-\mathrm{e}^{-2 \tau}\right)^{2}\right] \sin y \\
& +\frac{\lambda}{2} \mathrm{e}^{-2 \tau}\left(1-\mathrm{e}^{-2 \tau}\right) \sin (2 y) \\
& +\frac{\lambda^{2}}{8} \mathrm{e}^{-3 \tau}\left(1-\mathrm{e}^{-2 \tau}\right)^{2}\left(2+\mathrm{e}^{-2 \tau}\right) \sin (3 y)+O\left(\lambda^{3}\right) .
\end{aligned}
$$

In order to study how nonlinearity affects the damping rate we calculate the average over period energy of oscillations per unit 
area of the loop cross-section. In the leading-order approximation with respect to $\epsilon$ it is given by

$$
\begin{aligned}
E(\tau)=\epsilon^{2} \rho_{0} c_{\mathrm{s}}^{2} \mathcal{E}(\tau) & =\frac{\epsilon^{2} \omega}{4 \pi} \int_{0}^{2 \pi / \omega}\left(\rho_{0} u_{1}^{2}+\frac{c_{\mathrm{s}}^{2}}{\rho_{0}} \rho_{1}^{2}\right) \mathrm{d} t \\
& =\frac{\epsilon^{2} \rho_{0} c_{\mathrm{s}}^{2}}{\pi} \int_{0}^{2 \pi} f^{2}(\xi) \mathrm{d} \xi
\end{aligned}
$$

We define the dimensionless damping time $\tau_{\mathrm{d}}$ by the equation $\mathcal{E}\left(\tau_{\mathrm{d}}\right)=\mathrm{e}^{-2}$ (note that $\mathcal{E}(0)=1$ ). Substituting Eq. (52) in Eq. (53), we obtain

$\mathcal{E}(\tau)=\mathrm{e}^{-2 \tau}\left[1-\frac{\lambda^{2}}{4}\left(1-\mathrm{e}^{-2 \tau}\right)^{3}\right]+O\left(\lambda^{3}\right)$

Using this result we easily find

$\tau_{\mathrm{d}}=1-\frac{\lambda^{2}}{8}\left(1-\mathrm{e}^{-2}\right)^{3}+O\left(\lambda^{3}\right) \approx 1-0.081 \lambda^{2}$.

We see that nonlinearity reduces the damping time. This is an expected result because the nonlinearity transfers a part of the wave energy from the fundamental mode to the overtones that damp faster than the fundamental mode. As we have already pointed out, in the example considered by Ofman \& Wang (2002) $\lambda \approx 0.4$. Then, using Eq. (55), we find that the nonlinear damping time is by about $1.3 \%$ shoter than that given by the linear theory. We see that even for a moderately high value of $\lambda$ the correction to the damping time given by the nonlinear theory is very small.

We introduce the oscillation period $P=2 \pi / \omega=2 L / c_{\mathrm{s}}$ and the damping time

$t_{\mathrm{d}}=\frac{2 \gamma L^{2} \tau_{\mathrm{d}}}{\pi^{2}[\gamma \nu+(\gamma-1) \kappa]}$.

Then we obtain for the ratio of damping time to the wave period $t_{\mathrm{d}} / P=\tau_{\mathrm{d}} t_{\mathrm{dl}} / P$, where

$\frac{t_{\mathrm{dl}}}{P}=\frac{\gamma c_{\mathrm{s}} L}{\pi^{2}[\gamma \nu+(\gamma-1) \kappa]} \approx 1.3 \times 10^{-10} \frac{n_{0} L}{T_{0}^{2}}$,

where $t_{\mathrm{dl}}$ is the damping time given by linear theory, and $n_{0}$, $L$ and $T_{0}$ are measured in SI units. When deriving this formula we have used the relation $m=0.6 m_{\mathrm{p}}$. Using Eqs. (50) and (57) we obtain one more useful formula,

$\lambda \approx 4.2 \epsilon \frac{t_{\mathrm{dl}}}{P}$

In Fig. 1 the evolution of the velocity profile during one oscillation period is shown for $t_{\mathrm{dl}}=5 P$ and $\epsilon=0.1$, which corresponds to $\lambda \approx 2.1$. We can clearly see the wave steepening related to the generation of higher harmonics due do nonlinearity.

Figure 2 shows the dependence of the dimensionless damping time, $\tau_{\mathrm{d}}$, on the nonlinearity parameter $\lambda$. Once again we see that nonlinearity reduces the damping time. It is interesting to note that, although Eq. (55) was derived under the assumption that $\lambda \ll 1$, in fact it approximates $\tau_{\mathrm{d}}$ for $\lambda \lesssim 1$ fairly well.

We can see from Fig. 2 that the nonlinearity effect can be neglected for $\lambda \lesssim 1$. Then it follows from Eq. (58) that the nonlinear correction to the damping time must be taken into account only when $\epsilon t_{\mathrm{dl}} / P \gtrsim 0.3$. Once again using Fig. 2 we conclude that, for realistic values of $\lambda \lesssim 3$, nonlinearity can reduce the damping time by no more than about one third, so $t_{\mathrm{dl}} \lesssim 1.5 t_{\mathrm{d}}$.
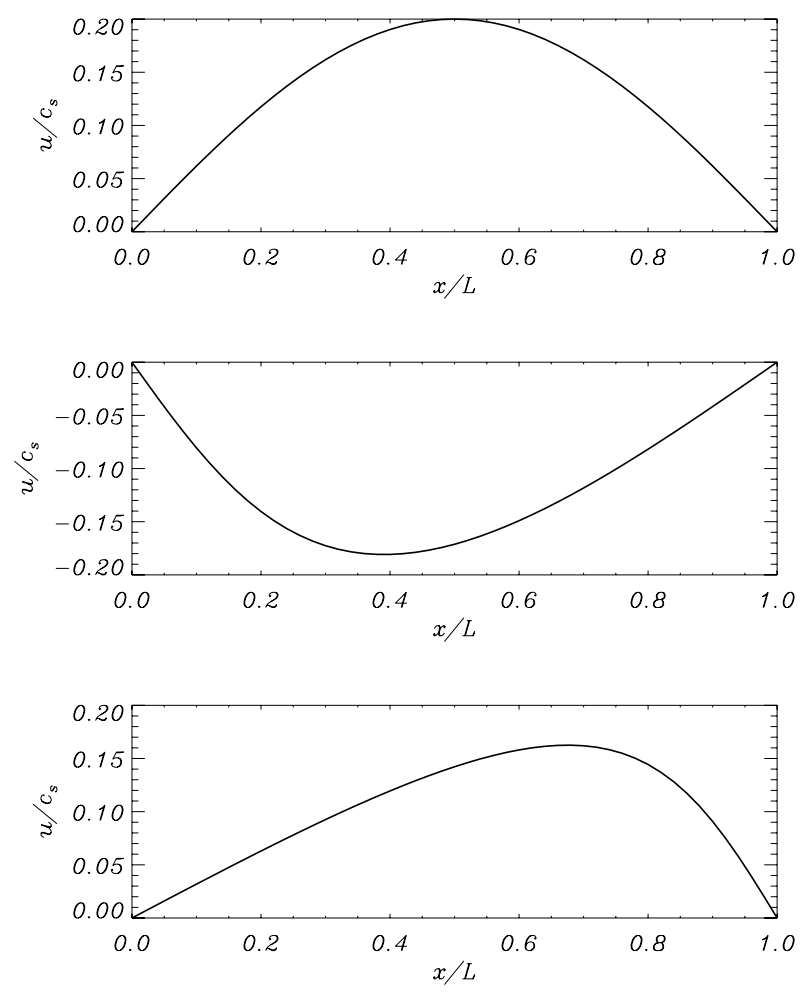

Fig. 1. Evolution of the velocity profile with time for $t_{\mathrm{dl}}=5 P$ and $\epsilon=0.1$. The upper, middle, and lower panel correspond to $t=0, P / 2$, and $P$ respectively.

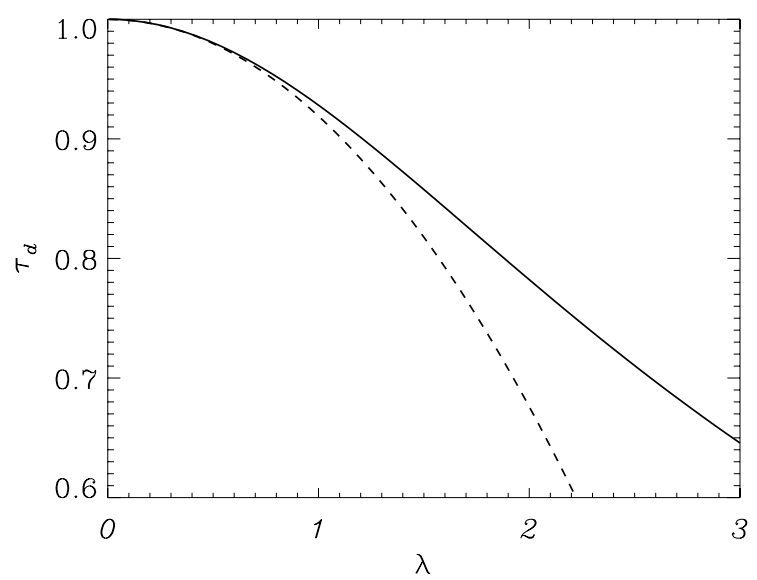

Fig. 2. Dependence of the dimensionless damping time $\tau_{\mathrm{d}}$ on the nonlinearity parameter $\lambda$. The solid line shows the dependence calculated numerically using the full Fourier expansion (49), while the dashed line shows the approximate dependence for small $\lambda$ given by Eq. (55).

Hence, eventually, the criterion for the importance of nonlinearity effect on the damping time is $\epsilon t_{\mathrm{d}} / P \gtrsim 0.2$. Since, in accordance with observations, $t_{\mathrm{d}} \lesssim 2 P$, we conclude that the nonlinearity effect is important only when $\epsilon \gtrsim 0.1$, i.e. when the Much number of the initial perturbation is of the order of or larger than 0.2 .

It is expedient to compare the results obtained in this paper with those presented by Verwichte et al. (2008). Unfortunately, we cannot carry out a quantitative comparison. The reason for this is that, for the loop parameters considered by Verwichte et al. (2008) Eq. (57) gives $t_{\mathrm{dl}} / P \approx 0.43$, so, for this set of loop parameters, the condition $t_{\mathrm{dl}} / P \gtrsim 1$ is not satisfied, i.e. one of the main assumptions made in this paper is violated. It follows 
from Fig. 5 of Verwichte et al. (2008) that the correct value is $t_{\mathrm{dl}} / P \approx 1.4$. This discrepancy is not surprising at all. It is well known that $t_{\mathrm{dl}} / P$ is inversely proportional to $k_{\|}$only when $k_{\|}$ is not too large. When $k_{\|}$increases, $t_{\mathrm{dl}} / P$ takes its minimum value, and then starts to grow (e.g. De Moortel \& Hood 2003). However, we can still make a qualitative comparison. First of all, similar to Verwichte et al. (2008), we found that nonlinearity reduces the damping time, which is a result expected on physical grounds. If, in accordance with the results obtain by Verwichte et al. (2008), we take $t_{\mathrm{dl}} / P \approx 1.4$, then, using Eq. (58), we have $\lambda \approx 6 \epsilon=3 V_{0} / c_{\mathrm{s}}$, where $V_{0}$ is the initial perturbation amplitude. Then it follows from Fig. 2 that the nonlinear correction to the damping time becomes important for $V_{0} / c_{\mathrm{s}} \gtrsim 0.3$, and for this values of $V_{0} / c_{\mathrm{s}}$ the nonlinear correction to the damping time is an almost linear function of $V_{0} / c_{\mathrm{s}}$. Taking into account that this correction is sufficiently small, we can conclude that the nonlinear correction to $P / t_{\mathrm{d}}$, which is proportional to $1 / \tau_{\mathrm{d}}$, is also an approximately linear function of $V_{0} / c_{\mathrm{s}}$. The inspection of Fig. 5 of Verwichte et al. (2008) reveals that $P / t_{\mathrm{d}}$ is also a linear function of $V_{0} / c_{\mathrm{s}}$ when $V_{0} / c_{\mathrm{s}}$ is approximately between 0.3 and 0.6 , i.e. for values of $V_{0} / c_{\mathrm{s}}$ that can be considered as moderate. Our analytical results are not applicable for higher values of $V_{0} / c_{\mathrm{s}}$. It is also worth noting that, for low values of $V_{0} / c_{\mathrm{s}}$, the dependence of $P / t_{\mathrm{d}}$ on $V_{0} / c_{\mathrm{s}}$ given by both Fig. 2 of this paper and by Fig. 5 of Verwichte et al. (2008) is approximately quadratic.

We now compare the results obtained in this paper with the dependence of the damping time on the initial perturbation amplitude obtained from the observations. This dependence is presented in Fig. 6 of Verwichte et al. (2008). If we approximate the solid curve in Fig. 2 by a linear curve, then we obtain $\tau_{\mathrm{d}} \approx 1-0.215 \lambda$, so $\tau_{\mathrm{d}}^{-1} \approx 1+0.215 \lambda$. Using this result yields

$\frac{P}{t_{\mathrm{d}}}=\frac{P}{\tau_{\mathrm{d}} t_{\mathrm{dl}}} \approx \frac{P}{t_{\mathrm{dl}}}(1+0.215 \lambda) \approx 0.73+0.44 \frac{V_{0}}{c_{\mathrm{s}}}$.

We see that the slope of the curve showing the dependence of $P / t_{\mathrm{d}}$ on $V_{0} / c_{\mathrm{s}}$ is about 0.44 , which is much smaller than the slope 1.8 found by Verwichte et al. (2008) from observations. Hence, similar to Verwichte et al. (2008), we conclude that our theory cannot explain the strong dependence of the damping time on the initial perturbation amplitude.

\section{Summary and conclusions}

We have studied the nonlinear damping of slow standing waves in hot coronal loops. We assumed that the wave amplitude is small and used it as a small parameter in the asymptotic expansions. In addition, we assumed that the wave damping is sufficiently slow and introduced the "slow" time related to the evolution of the wave amplitude and shape. We showed that, in the leading-order approximation, the nonlinear standing wave is a superposition of two identical nonlinear waves propagating in the opposite directions. The evolution of the two waves is governed by the Burgers equation.

When written in the dimensionless variables, the Burgers equation contains only one dimensionless parameter $\lambda$ that defines the ratio of the nonlinear and dissipative term. Using the Cole-Hopfe substitution, we obtained an analytical solution describing the evolution of a standing wave that has the form of the linear fundamental eigenmode at the initial moment of time. Then we used this solution for the parametric study of the nonlinear damping of standing waves. In particular, we studied the dependence of $t_{\mathrm{d}} / t_{\mathrm{dl}}$ on $\lambda$, where $t_{\mathrm{d}}$ and $t_{\mathrm{dl}}$ are the damping time given by linear and nonlinear theory, respectively. We showed that, for $\lambda \leq 3$, nonlinearity can reduce the damping time by no more than about one third. Moreover, for realistic coronal loop parameters and observed periods and damping times, the nonlinearity effect on the damping time must be taken into account only when the Mach number of the initial velocity perturbation is sufficiently large, of the order of or larger than 0.2.

We compared the results obtained in this paper with those obtained from observations. Similar to Verwichte et al. (2008), we found that out theory cannot explain strong dependence of the damping time on the initial perturbation amplitude.

Finally, we note that the analysis presented in this paper can be modified to study the effect of loop cooling on the nonlinear damping of slow standing waves in hot coronal loops. Recently, this effect has been studied in the linear approximation by Al-Ghafri \& Erdélyi (2013).

Acknowledgements. M.S.R. acknowledges the support by the STFC grant, and thanks the unknown referee for very useful comments.

\section{References}

Abramowitz, M., \& Stegun, I. 1964, Handbook of Mathematical Functions (National Bureau of Standards)

Al-Ghafri, K. S., \& Erdélyi, R. 2013, Sol. Phys., 283, 413

Bender, C. M., \& Orszag, S. A. 1978, Advanced Mathematical Methods for Scientists and Engineers (New York: McGraw-Hill)

Braginskii, S. I. 1965, Rev. Plasma Phys., 1, 205

Burgers, J. M. 1948, in Advances in Applied Mechanics, eds. R. von Mises, \& T. von Karman (New York, N. Y.: Academic Press Inc.), 171

Cole, J. D. 1951, Quart. Appl. Math., 9, 225

De Moortel, I., \& Hood, A. W. 2003, A\&A, 408, 755

Goedbloed, J. P. H., \& Poedts, S. 2004, Principles of Magnetohydrodynamics (Cambridge, UK: Cambridge University Press)

Gusev, V. E. 1984a, Soviet Physics Acoustics-USSR, 30, 121

Gusev, V. E. 1984b, Vestnik Moskovskogo Universiteta Seriya 3 Fisika Astronomiya, 25, 29

Hopf, E. 1950, Comm. Pure and Appl. Math., 3, 201

Kliem, B., Dammasch, I. E., Curdt, W., \& Wilhelm, K. 2002, ApJ, 568, L61

Mendoza-Briceño, C. A., Erdélyi, R., \& Sigalotti, L. D. G. 2004, ApJ, 605, 493

Ofman, L., \& Wang, T. 2002, ApJ, 580, L85

Priest, E. 1982, Solar Magneto-Hydrodynamics, Geophysics and Astrophysics Monographs (Kluwer Academic Publishers)

Rudenko, O. V., \& Solujan, S. I. 1977, Theoretical Foundations of Nonlinear Acoustics (New York and London: Plenum Publishing Corporation, Consultants Bureau)

Ruderman, M. S., \& Nocera, L. 1998, A\&A, 340, 287

Sigalotti, L. D. G., Mendoza-Briceño, C. A., \& Luna-Cardozo, M. 2007, Sol. Phys., 247, 187

Spitzer, L. J. 1962, Physics of Fully Ionized Gases (New York: Wiley Interscience)

Verwichte, E., Haynes, M., Arber, T. D., \& Brady, C. S. 2008, ApJ, 685, 1286

Wang, T. J. 2011, Space Sci. Rev., 158, 397

Wang, T. J., Solanki, S. K., Curdt, W., Innes, D. E., \& Dammasch, I. E. 2002, ApJ, 574, L101

Wang, T. J., Solanki, S. K., Curdt, W., et al. 2003a, A\&A, 406, 1105

Wang, T. J., Solanki, S. K., Innes, D. E., Curdt, W., \& Marsch, E. 2003b, A\&A, 402, L17

Whitham, G. B. 1974, Linear and Nonlinear Waves (John Wiley \& Sons) 